\title{
Whole grain intake and pancreatic cancer risk
}

\author{
Glenn A. Gaesser \\ College of Health Solutions, Arizona State University, Phoenix, AZ, USA \\ Correspondence to: Glenn A. Gaesser, PhD. College of Health Solutions, Arizona State University, 550 N. Third St., Phoenix, AZ 85004, USA. Email: \\ Glenn.gaesser@asu.edu. \\ Comment on: Schacht SR, Olsen A, Dragsted LO, et al. Whole-Grain Intake and Pancreatic Cancer Risk-The Danish, Diet, Cancer and Health \\ Cohort. J Nutr 2021;151:666-74.
}

Submitted Jun 02, 2021. Accepted for publication Jun 25, 2021.

doi: $10.21037 / \mathrm{hbsn}-21-221$

View this article at: https://dx.doi.org/10.21037/hbsn-21-221

Cancer is one of the leading causes of death worldwide, and there is evidence that dietary factors contribute significantly to cancer risk (1). Whole grain foods are associated with a lower risk of several chronic diseases, including cancer, and are recommended as part of a healthy diet $(1,2)$. Over the past 10 years, multiple meta-analyses have shown that whole grain intake is associated with lower risk of total and sitespecific cancer incidence and mortality (3). Results from these studies indicate that in categorical analyses of highest vs. lowest intakes of whole grains, a high consumption of whole grains is associated with a $6 \%$ to $12 \%$ lower risk of cancer mortality. Dose-response analyses demonstrated that each 30 grams/day increase in whole grain intake is associated with an approximate $7 \%$ lower risk of cancer mortality.

For site-specific cancers, meta-analyses have demonstrated that whole grain intake is associated with lower risks of colorectal cancer, gastric cancer, and pancreatic cancer (3). When comparing highest $v$ s. lowest consumption groups, high intake of whole grains is associated with an $11 \%$ to $21 \%$ lower risk of colorectal cancer, with each 90 grams/day intake corresponding to a $15 \%$ to $17 \%$ lower risk of colorectal cancer. For gastric and pancreatic cancer, meta-analyses indicate that high intake of whole grains is associated with a $13 \%$ to $39 \%$ lower risk of gastric cancer and a $24 \%$ to $30 \%$ lower risk of pancreatic cancer. No dose-response meta-analyses have been published on the association between whole grain intake and either gastric or pancreatic cancer. Another limitation is that the meta-analyses on gastric and pancreatic cancer relied almost entirely on case-control studies, whereas most of the meta-analyses on colorectal cancer used almost exclusively prospective cohort studies (3).

The recent publication by Schacht et al. (4) provides new insight on the association between whole grain intake and pancreatic cancer risk (4). They examined the association between whole grain intake and pancreatic cancer risk in 57,053 middle-aged adults participating in the Danish Diet, Cancer and Health cohort, with approximately equal percentages of men and women. After a mean follow-up of 17.5 years, an inverse association between whole grain intake and pancreatic cancer risk was found (Figure 1). Each 50 grams/day of whole grain foods was associated with a $7 \%$ lower risk of pancreatic cancer [hazard ratio $(\mathrm{HR})=0.93$; 95\% confidence interval (CI): $0.85-1.01$ ]. In categorical analyses, a $25 \%$ lower risk was observed when comparing highest vs. lowest intake quartiles (HR $=0.75$; $95 \% \mathrm{CI}$ : $0.57-1.04)$. In dose-response analyses, the HR ranged from 0.90 to 0.93 , depending on the number of adjustments included in the model (age, educational level, smoking, physical activity, alcohol, diabetes, body mass index, and total energy intake), with the upper boundary of the $95 \%$ CI between 0.97 and 1.01. For the categorical analyses, the HR ranged from 0.68 to 0.76 , depending on adjustments, with the upper boundary of the $95 \%$ CI between 0.90 to 1.04. The data presented in Figure 1 are for the model which adjusted for all potential confounders.

Even though the HRs in categorical analyses by Schacht et al. were not always statistically significant (i.e., the upper boundary of the $95 \% \mathrm{CI}>1.00$ when the model included adjustments for BMI, diabetes and total energy intake), the HR range of $0.68-0.76$ was very similar to the lower odds ratios reported in the two published meta-analyses on the association between whole grain intake and pancreatic 


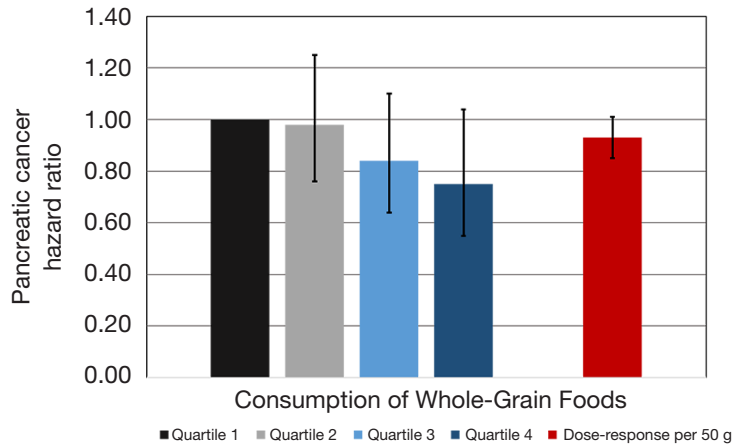

Figure 1 Association between whole-grain food intake and pancreatic cancer incidence among 57,053 adults in the Danish Diet, Cancer and Health cohort. Quartile 1 = referent group. Hazard ratios for quartiles 2-4 and the dose-response include \pm $95 \%$ confidence intervals. Hazard ratio for the dose-response is for each 50 grams/day intake of whole-grain foods.

cancer risk (Figure 2) $(5,6)$. The meta-analysis by Jacobs et al. (5) produced an odds ratio of 0.70 (95\% CI: 0.54-0.91), and the meta-analysis by Lei et al. (6) produced an odds ratio of 0.76 (95\% CI: $0.64-0.91$ ). It is important to note that all but one of the publications included in these two meta-analyses were case-control studies, and the only cohort study included only male smokers in Finland. Thus, the recent study by Schacht $e t$ al. is the first and only cohort study on whole grain intake and pancreatic cancer risk that included both men and women.

In this Danish cohort, the major source of whole grain intake was rye bread, which comprised $88 \%$ of the whole grain intake. Moreover, when examining specific whole grain foods, only rye bread consumption was associated with lower risk of pancreatic cancer. Thus, generalizing to populations in which rye consumption is much lower must be viewed with caution. However, the HRs for wheat and whole grain bread were comparable to that of rye, even if not always statistically significant. Also, most of the studies included in the two published meta-analyses included wheat and whole-grain bread as primary whole grain exposures $(5,6)$.

An interesting finding in the recent study by Schacht $e t$ al. is that the lower pancreatic cancer risk associated with consumption of whole-grain foods was only observed in men (HR $=0.90 ; 95 \%$ CI: 0.81-0.99) and not in women (HR $=0.98,95 \%$ CI: $0.87-1.10)$. Reasons for this are not clear, but could possibly be due in part to the lower consumption of rye among women in this cohort. Nonetheless, because

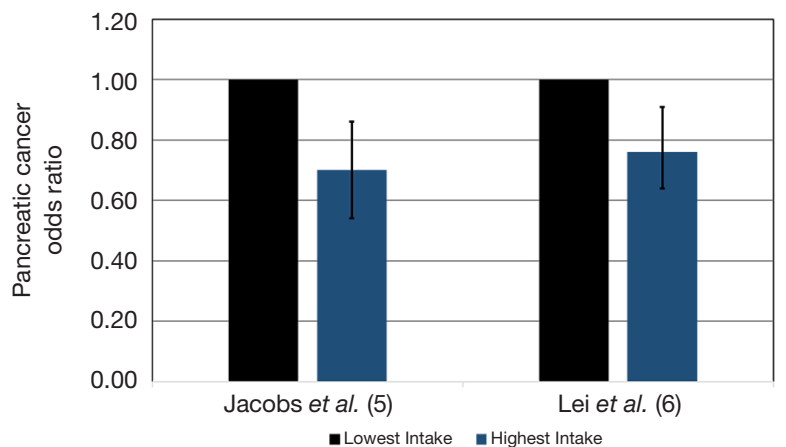

Figure 2 Association between whole grain intake and pancreatic cancer risk in two meta-analyses. Odds ratios for the highest intake groups include $\pm 95 \%$ confidence intervals.

the lower cancer risk was only observed in men, and the only previously published cohort study included only male smokers, there has yet to be a cohort study to show that whole grain intake is associated with reduced pancreatic cancer risk in women. This requires additional research to clarify whether a true sex difference exists.

\section{Potential mechanisms}

In 2000, the American Association of Cereal Chemists defined whole grains as consisting of the "intact, ground, cracked or flaked caryopsis, whose principal anatomical components-the starchy endosperm, germ and branare present in the same relative proportions as they exist in the intact caryopsis" (7). This definition has been adopted by regulatory organizations to support recommendations for greater consumption of whole-grain products (8). Refined grains have some or all of the bran layers removed during the milling process, which diminishes the amount of fiber and micronutrients (8). This may explain why whole grain intake has been shown to be associated with lower overall and site-specific cancer risk whereas refined grain consumption has not $(3,9)$.

Whole grains may reduce cancer risk via several mechanisms $(10,11)$. Whole grain intake is correlated with cereal fiber intake (12), and cereal fiber consumption is associated with lower cancer risk (13). Other aspects of whole grains may also contribute to the reduced risk because whole grain intake is associated with reduced cancer risk even after adjusting for cereal fiber intake (14). Consequently, the lower risk of total and cite-specific cancers associated with whole grain intake could be due to other properties of whole 
grains. Whole grains are an important source of antioxidants, phenolic acids and phytoestrogens, which can reduce oxidative damage in cells (10). Also, whole grains contain numerous bioactive compounds that have anticarcinogenic properties $(10,11)$. The synergetic effects of these bioactive compounds-the "whole grain package" (10)—likely explain the consistent findings from cohort studies on the reduced cancer risk associated with whole grain intake.

\section{Public health implications}

Consumption of whole grains is central to public health recommendations to reduce cancer risk $(1,2)$. For example, the United States dietary guidelines recommend consuming at least 3 servings per day of whole grains, with a serving defined as 1 ounce-equivalent ( 28 grams) of a whole grain product (2). Less than $7 \%$ of the U.S. population achieves this goal, with mean whole grain intake $<1$ serving per day (15). In fact, $70 \%$ of the U.S. population consumes less than 1 serving of whole grains per day (15). Thus, meeting minimum recommendations for whole grain intake could lead to meaningful reductions in cancer risk in the general population. Perhaps more importantly, in view of the doseresponse between whole grain intake and cancer mortality (3) and pancreatic cancer incidence (4), virtually any increase in whole grain intake may help reduce cancer risk.

\section{Acknowledgments}

Funding: None.

\section{Footnote}

Provenance and Peer Review: This article was commissioned by the editorial office of Hepatobiliary Surgery and Nutrition. The article did not undergo external peer review.

Conflicts of Interest: The author has completed the ICMJE uniform disclosure form (available at https://hbsn. amegroups.com/article/view/10.21037/hbsn-21-221/coif). The author has no conflicts of interest to declare.

Ethical Statement: The author is accountable for all aspects of the work in ensuring that questions related to the accuracy or integrity of any part of the work are appropriately investigated and resolved.

Open Access Statement: This is an Open Access article distributed in accordance with the Creative Commons Attribution-NonCommercial-NoDerivs 4.0 International License (CC BY-NC-ND 4.0), which permits the noncommercial replication and distribution of the article with the strict proviso that no changes or edits are made and the original work is properly cited (including links to both the formal publication through the relevant DOI and the license). See: https://creativecommons.org/licenses/by-nc-nd/4.0/.

\section{References}

1. World Cancer Research Fund and American Institute for Cancer Research. Diet, Nutrition, Physical Activity and Cancer: a Global Perspective 2018 [Accessed May 25, 2021]. Available online: https://www.wcrf.org/diet-andcancer/.

2. U.S. Department of Agriculture and U.S. Department of Health and Human Services. Dietary Guidelines for Americans, 2020-2025. 9th Edition 2020 [Accessed May 25, 2021]. Available online: https://www. DietaryGuidelines.gov.

3. Gaesser GA. Whole Grains, Refined Grains, and Cancer Risk: A Systematic Review of Meta-Analyses of Observational Studies. Nutrients 2020;12:3756.

4. Schacht SR, Olsen A, Dragsted LO, et al. Whole-Grain Intake and Pancreatic Cancer Risk-The Danish, Diet, Cancer and Health Cohort. J Nutr 2021;151:666-74.

5. Jacobs DR Jr, Marquart L, Slavin J, et al. Whole-grain intake and cancer: an expanded review and meta-analysis. Nutr Cancer 1998;30:85-96.

6. Lei Q, Zheng H, Bi J, et al. Whole Grain Intake Reduces Pancreatic Cancer Risk: A Meta-Analysis of Observational Studies. Medicine (Baltimore) 2016;95:e2747.

7. AACC Committee to Define Whole Grain. AACC members agree on definition of whole grain. Cereal Foods World 2000;45:79.

8. Jones JM, Garcia CG, Braun HJ. Perspective: Whole and Refined Grains and Health-Evidence Supporting "Make Half Your Grains Whole". Adv Nutr 2020;11:492-506.

9. Gaesser GA. Perspective: Refined Grains and Health: Genuine Risk, or Guilt by Association? Adv Nutr 2019;10:361-71.

10. Fardet A. New hypotheses for the health-protective mechanisms of whole-grain cereals: what is beyond fibre? Nutr Res Rev 2010;23:65-134.

11. Slavin J. Whole grains and human health. Nutr Res Rev 2004;17:99-110.

12. McKeown NM, Yoshida M, Shea MK, et al. Whole-grain 
intake and cereal fiber are associated with lower abdominal adiposity in older adults. J Nutr 2009;139:1950-5.

13. Hajishafiee M, Saneei P, Benisi-Kohansal S, et al. A. Cereal fibre intake and risk of mortality from all causes, CVD, cancer and inflammatory diseases: a systematic review and meta-analysis of prospective cohort studies. Br J Nutr 2016;116:343-52.

14. Huang T, Xu M, Lee A, et al. Consumption of whole

Cite this article as: Gaesser GA. Whole grain intake and pancreatic cancer risk. HepatoBiliary Surg Nutr 2021;10(4):530533. doi: 10.21037/hbsn-21-221 grains and cereal fiber and total and cause-specific mortality: prospective analysis of 367,442 individuals. BMC Med 2015;13:59.

15. O'Neil CE, Nicklas TA, Zanovec M, et al. Whole-grain consumption is associated with diet quality and nutrient intake in adults: the National Health and Nutrition Examination Survey, 1999-2004. J Am Diet Assoc 2010;110:1461-8. 